\title{
Nota técnica \\ ANÁLISIS FINANCIERO PARA LA IMPLEMENTACIÓN DE UN SISTEMA ESTABULADO EN UNA FINCA DE GANADO DE LECHE EN COSTA RICA
}

\author{
Luis Villalobos $^{1 / *}$, Leonardo Rivera* \\ Palabras clave: Factibilidad, estabulación, ganado de leche, análisis marginal, VAN. \\ Keywords: Feasibility, feedlot, dairy cattle, marginal analysis, NPV. \\ Recibido: 01/03/12 \\ Aceptado: 24/08/12
}

\begin{abstract}
RESUMEN
Se evaluó la viabilidad financiera para la implementación de un sistema de producción de ganado de leche estabulado, en una finca que utiliza un sistema semiestabulado. La evaluación se realizó por medio del análisis de las áreas funcionales de la empresa (producción, mercadeo, recursos humanos y finanzas), con el fin de determinar la situación técnica previa a un cambio en el sistema productivo, y del uso de registros productivos y reproductivos en el programa Invest-GA® para el flujo de hato. El análisis financiero consistió en la búsqueda de información de mercado, estimación de la capacidad de endeudamiento de la empresa y elaboración de flujos de caja nominales a partir de registros financieros del programa DairyProfit@. Con los diferentes escenarios proyectados se obtuvo el valor actual neto (VAN) como indicador financiero. Se realizó un análisis de sensibilidad unidimensional con variaciones en el nivel productivo individual de los animales y un análisis marginal por medio del VAN incremental mostrado por los diferentes escenarios, comparado con el desarrollo de la finca sin realizar inversiones. El escenario que mostró ser más viable fue la inversión en la construcción de instalaciones para estabulación, compra de un carro mezclador
\end{abstract}

1 Autor para correspondencia. Correo electrónico: luis.villalobosvillalobos@ucr.ac.cr

\section{ABSTRACT}

Financial analysis for the implementation of a feedlot in a dairy cattle farm in Costa Rica. The financial feasibility for the implementation of a feedlot in a dairy farm was evaluated. The evaluation was made in the functional departments (production, marketing, human resources and finances) to determine the technical situation before changing the productive system. The reproductive and productive records were used to map out the growing of the herd with the program Invest$\mathrm{GA} \otimes$. Financial analysis was carried out by searching for market information, estimating the borrowing capacity of the company and nominal cash flows made from financial records of the DairyProfit ${ }^{\circledR}$ program. The net present value (NPV) was obtained as a financial indicator from the different projections. A sensitivity analysis was made varying the production level of the animals (one-dimensional), and a marginal analysis was made by using the incremental NPV obtained in the projections versus the productive system without investments. The best projection for the dairy farm was the investment in a stable, a mixer and receiving a lower payment for the additional milk delivered to the factory obtaining $\phi 237.129 .530,15$ and $\phi 89.169 .075,85$ for the NPV

\footnotetext{
* Escuela de Zootecnia. Centro de Investigaciones en Nutrición Animal. Universidad de Costa Rica. San José, Costa Rica.
} 
y no adquirir certificados para la entrega de leche con un pago diferenciado que presentó un VAN de $\phi 237.129 .530,15$ y un VAN incremental de $\phi 89.169 .075,85$. El recibo de producto en las plantas industrializadoras lácteas es un factor determinante para la implementación de sistemas más intensivos que permitan aumentar la producción de leche en las fincas de Costa Rica. La productividad en las fincas lecheras determina su competitividad en el mercado, ya que conforme se presenta una mayor especialización en los sistemas productivos, existen más factores externos que pueden afectar su desempeño y por tanto, los sistemas de control interno deben ajustarse a la situación actual.

\section{INTRODUCCIÓN}

En los estudios de proyectos se tiende a anteponer la rentabilidad como único criterio de aceptación o rechazo, sin embargo éstos deben ser una herramienta que permita contar con más información al encargado de tomar la decisión (Sapag y Sapag 2008).

La factibilidad económica de cualquier cambio que se vaya a realizar en los sistemas productivos pecuarios permite tener un panorama más completo sobre la realidad de la operación y son un complemento al análisis técnico que se realiza como parte de un enfoque más integral en especies como ganado de leche, cabras y ovejas (Campos 2005, Delgado 2008, Vásquez 2011). De acuerdo con Sapag y Sapag (2008) el análisis completo de cualquier proyecto, ya sea en ejecución o nuevo, requiere al menos de 4 estudios: de mercado, técnico, organizacional-administrativolegal y financiero.

El aumento en el consumo de productos lácteos a nivel mundial se ha debido a que los consumidores cada vez adquieren mayor conciencia de las propiedades benéficas que estos presentan y a que el sector industrial ha logrado and incremental NPV, respectively. The receiving of the product by the dairy factories is one of the main factors affecting the implementation of more intensive systems in the dairy farms of Costa Rica. Productivity of dairy farms determines their competitiveness, because as the specialization in dairy production systems grows, there are more external factors that may affect performance and, therefore, internal control systems must be accurate to adapt to the current situation.

una expansión constante por medio de investigación y desarrollo, con nuevas tecnologías y la incursión en el mercado con productos novedosos y funcionales (Comisión de las Comunidades Europeas 2009).

Debido a la presión demográfica y al aumento en el precio de la tierra a causa de su utilización para fines no agrícolas (urbanización y turismo principalmente), los sistemas productivos cada vez deben ser más eficientes en el uso de los recursos disponibles y disminuir la dependencia a insumos externos con el objetivo de que el costo de producción sea menor y permita ser competitivo a nivel internacional (Villegas 2007b). De acuerdo con lo anterior es que se justifica la implementación de sistemas productivos que hagan un uso más intensivo de los terrenos (cultivos) y que necesariamente involucre un aumento en la capacidad productiva de la finca por medio del aumento en la carga animal (vacas en ordeño) antes que el aumento individual en la producción de leche.

El objetivo de la presente investigación fue determinar la factibilidad del cambio de un sistema de producción de leche semiestabulado a uno estabulado. 


\section{MATERIALES Y MÉTODOS}

El presente estudio se realizó en una finca comercial productora de leche ubicada en el distrito de Santa Rosa, Oreamuno, Cartago, ubicado en la sección Oriental del Valle Central a una altitud de 2000 msnm. Dicha zona tiene influencia climática del Caribe y se clasifica como bosque húmedo tropical premontano (Holdridge 1947, citado por Janzen 1991), su precipitación promedio anual es de $2239 \mathrm{~mm}$, la temperatura mínima y máxima promedio son $13,4^{\circ} \mathrm{C}$ y $23,3^{\circ} \mathrm{C}$ respectivamente y presentan pocas variaciones durante el año (Retana 2011).

La finca cuenta con ganado de la raza Holstein de alto potencial genético bajo un sistema semiestabulado en el cual los animales en producción duermen en un corral o galerón después del ordeño de la tarde donde se les brinda alimento para la noche (12 horas) y posterior al ordeño de la mañana son enviados a un potrero en el que pastorean durante el día hasta el siguiente ordeño.

\section{Búsqueda de precios actualizados de las inversiones a realizar}

Se llevó a cabo una recolección de datos sobre los precios de las inversiones más significativas a realizar en la finca con el fin de obtener una adecuada estimación de las inversiones. Dicha información se recolectó en casas comerciales de venta de equipo así como la información suministrada por la finca y fuentes directas por medio de los costos del $\mathrm{m}^{2}$ de construcción que maneja el Colegio de Ingenieros y Arquitectos. Debido a que la empresa requiere producir y entregar más leche, en su momento requeriría invertir en la compra de certificados de aportación de la Cooperativa de Productores de Leche R.L. (COPROLE R.L.).

\section{Búsqueda de información de entidades financieras}

Se recolectó información de las entidades que el productor utilizaría como fuente de financiamiento, específicamente en Coopelecheros ya que la empresa puede acceder a crédito por ser asociada a COPROLE R.L. Para esto se realizaron estimaciones de las inversiones necesarias $\mathrm{y}$ acorde con los montos, se diagnosticaron las posibilidades reales de endeudamiento para el productor.

Al momento del estudio, Coopelecheros ofrecía una tasa de interés del $13 \%$ para la construcción del corral, carro mezclador, sistema de riego y sala de ordeño y de $14 \%$ para la compra de certificados de aportación, éstas serían las inversiones a realizar en la operación. Los créditos serían a un plazo de 10 años de cancelación y sin cálculo de costo de capital por tratarse de financiamiento externo.

\section{Análisis técnico y financiero}

Para determinar la situación técnica bajo el sistema actual, se hizo un análisis de Fortalezas, Oportunidades, Debilidades y Amenazas (FODA) a partir de 4 áreas funcionales de la empresa: producción, finanzas, mercadeo y recursos humanos, asignándole un nivel de importancia del 1 al 3 a cada aspecto de las distintas áreas y ponderándolos, con valores negativos en las debilidades y amenazas y positivos en fortalezas y oportunidades, con esto se realizó un balance para cada área y se determinó en cuales aspectos se debía priorizar esfuerzos.

Se analizaron 3 diferentes razones financieras de endeudamiento con las fórmulas de Salas (2005) para respaldar las posibilidades reales ante una solicitud externa:

- $\quad$ Razón de deuda: (Pasivo total/Activo total) x 100 .

- Razón pasivo-aportación: (Pasivo total/ Capital total) x 100 .

Cobertura de interés: (Utilidad operativa/ Gastos financieros).

Se analizó el proyecto bajo el sistema actual de manejo con un hato en producción constante tal como se muestra en el Cuadro 1, así como con la implementación de un sistema estabulado y con la inclusión de las inversiones necesarias (año 
Cuadro 1. Composición promedio del hato en el momento del estudio según el número de lactancias.

\begin{tabular}{ccc}
\hline Número de lactancias & Cantidad & $\%$ \\
\hline 0 & 46 & 40,17 \\
1 & 20 & 17,29 \\
2 & 19 & 16,50 \\
3 & 10 & 8,93 \\
4 & 6 & 4,81 \\
5 & 6 & 4,84 \\
6 & 4 & 3,59 \\
7 & 2 & 2,11 \\
8 & 2 & 1,50 \\
9 & 1 & 0,85 \\
\hline
\end{tabular}

0) para su operación mediante una ampliación de su capacidad productiva, y el análisis del valor actual neto (VAN) a un plazo de 10 años, en el caso de la proyección sin inversiones se mantuvo el hato constante. Para las proyecciones se utilizó como base los estados financieros de los últimos 5 períodos fiscales y reportes financieros del programa DairyProfit@ (IAP-SOFT 2007); el mismo programa contabiliza los costos relativos por su tratamiento y, su efecto en la productividad de la finca, se manifiesta en la proyección realizada en el programa Invest-GA® (IAP-SOFT
2008), el cual utilizó los parámetros técnicos (productivos y reproductivos) a partir de la base de datos de la finca (Cuadro 2).

En términos reproductivos, la finca cuenta con sistemas de sincronización de celos con el objetivo de aumentar la precisión en su diagnóstico y al mismo tiempo hacer una selección constante de los animales y descartar los que presenten problemas de fertilidad (Cuadro 2). El costo de producción de leche para la finca fue de $\phi 219,92$ equivalentes a $\$ 0,395$ (TC $1 \$=\phi 554$ ), valor menor al precio recibido por pago de leche

Cuadro 2. Parámetros productivos y reproductivos de la empresa en el momento del estudio.

\begin{tabular}{|c|c|}
\hline Parámetro & Valor \\
\hline Animales paridos (se incluyen abortos) & 52 \\
\hline Servicios por concepción & 1,36 \\
\hline Servicios por animal servido & 1,68 \\
\hline Intervalo entre partos & 405 \\
\hline Descarte por problemas de fertilidad (animales) & 2 \\
\hline Edad a primer parto (meses) & 30,7 \\
\hline Animales con más de 36 meses (\%) & 7,1 \\
\hline Vacas adultas & 68 \\
\hline Vacas en ordeño & 56 \\
\hline Reemplazos & 47 \\
\hline Producción láctea promedio $\left(\mathrm{kg} . \mathrm{vaca}^{-1}\right)$ & 20,3 \\
\hline Producción láctea mensual $\left(\mathrm{kg} \cdot \mathrm{mes}^{-1}\right)$ & 34243 \\
\hline Producción de sólidos totales (kg.mes ${ }^{-1}$ ) & 4067 \\
\hline Costo de producción de leche (colones. $\mathrm{kg}^{-1}$ ) & 219,92 \\
\hline Producción anual láctea (kg.ha-1 pastoreo) & 10090 \\
\hline Relación vacas adultas empleado & 10,8 \\
\hline Carga animal (animales.ha ${ }^{-1}$ ) & 2,8 \\
\hline
\end{tabular}


$\left(\notin 266 . \mathrm{kg}^{-1}\right)$, por esto en parte la finca decidió aumentar su capacidad productiva por medio de inversiones que considera estratégicas para entregar un mayor volumen de leche. La producción individual fue de $20 \mathrm{~kg} . v a c a^{-1}$, dicho indicador tiene importancia posteriormente en el análisis de sensibilidad como parte de la respuesta medible en los animales como resultado del cambio en el sistema de producción, asimismo la producción anual láctea es un indicador de eficiencia en el uso del área disponible en la finca, de igual forma la producción de sólidos, la relación vacas adultas empleado y la carga animal se verían aumentadas con la tecnificación del sistema productivo.

\section{Análisis de sensibilidad y marginal}

Los diferentes escenarios propuestos fueron: situación actual, inversiones menores como sistema de riego, sala y equipo de ordeño sin adquirir certificados e inversiones mayores como corral estabulación, carro mezclador con certificados; (Sapag y Sapag 2008). Para cada uno de los escenarios se estimó el valor actual neto, esto debido a que el proyecto consistió en inversiones que se debían realizar para aumentar la productividad de un sistema productivo en operación.

Para el análisis de sensibilidad unidimensional se escogió la situación en la cual la empresa realizaría una inversión mayor (corral, carro mezclador y compra de certificados de aportación). En la simulación se modificó el nivel productivo individual en $2 \mathrm{~kg}$ de leche hacia arriba (Optimista) o hacia abajo (Pesimista) con base en el promedio de producción de los animales (Normal). Se llevó a cabo un análisis marginal a través del VAN incremental, el cual consiste en la diferencia entre el VAN con el proyecto y el VAN sin el proyecto para determinar la viabilidad de implementarse con respecto a la situación actual proyectada. Este se realizó para cada una de las situaciones proyectadas, así como en los escenarios propuestos para el análisis de sensibilidad de la empresa.

\section{RESULTADOS Y DISCUSIÓN}

\section{Situación técnica y financiera de la finca bajo el sistema de producción actual (semiestabulado)}

Normalmente el hato de la finca se compone de 50 a 63 vacas en producción, 17 vacas secas y 41 animales que serán los futuros reemplazos (varía en la cantidad de terneras y novillas a empadre). En la Figura 1 se muestra el comportamiento de crecimiento del hato por medio de

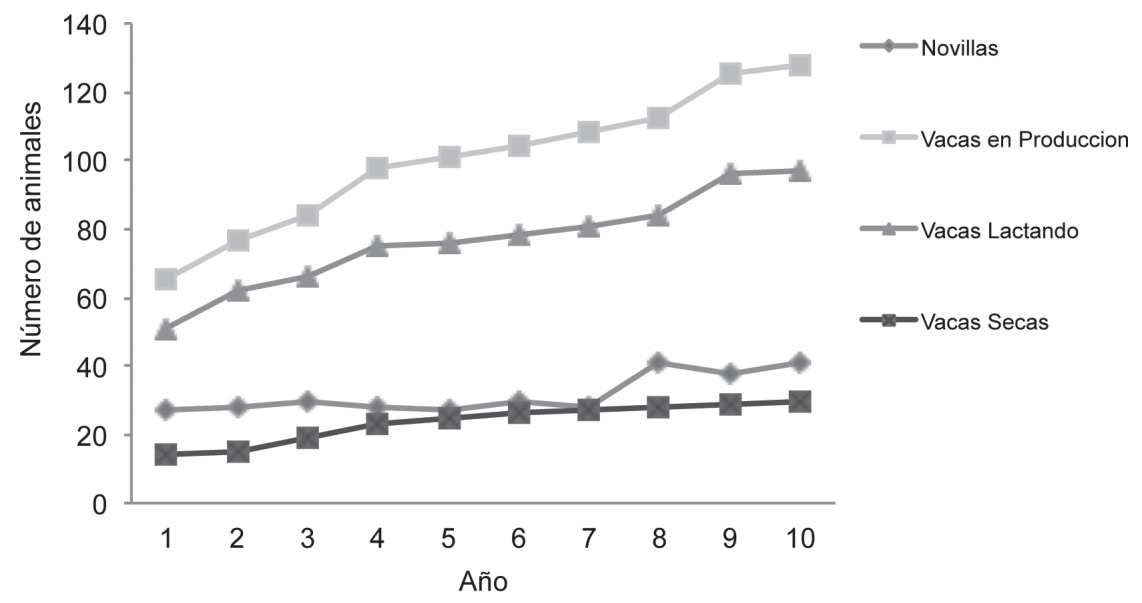

Fig. 1. Flujo de crecimiento del hato con base en una simulación estocástica llevada a cabo en el programa Invest-GA en 10 años. 
una simulación en el programa Invest-GA® con el uso los registros y parámetros técnicos de la finca como referencia.

Como parte de las inversiones menores a realizar en la finca está la implementación de un sistema de manejo de aguas de lavado de la lechería durante el primer año de transición que corresponde al año 0 y al sistema de producción estabulado. Esta inversión es parte de los requisitos de operación que actualmente solicita el Servicio Nacional de Salud Animal (SENASA) para entregar el certificado veterinario de operación (CVO) (SENASA 2006). Asimismo, se podrá contar con un sistema de distribución de los purines en la finca debido a la necesidad de contar con mayor área cultivable para la producción de forrajes (R. Salas 2008. Comunicación personal).

En términos económicos se esperaba una respuesta del sistema de manejo de aguas de lavado por medio del ahorro en compras de fertilizantes químicos; asimismo el riego constante de los potreros permitiría que la producción del pasto se vea menos afectada por cambios estacionales con lo cual se disminuirían las compras de fuentes forrajeras como el heno. La utilización de agua para lavado del corral sería menor, ya que se estimó en 90001 que se gastan en 2 lavados por día, por lo que con el sistema actual dicha cantidad se puede reducir a la mitad y aprovechar los purines de 60 animales en producción para su riego en los potreros.

La empresa estudiada cuenta con capacidad de entregar alrededor de $1481 \mathrm{~kg}$ de leche por día a la empresa industrializadora (10 $367 \mathrm{~kg}$ semanales) y el deseo de aumentar su producción fue por motivo de una alta demanda del producto en el momento de realizado este proyecto y la apertura de las empresas industrializadoras a nivel nacional a recibir más producto, sin embargo el excedente que se entregue recibe normalmente un pago diferenciado equivalente a un $90 \%$ del precio normal de entrega que para la empresa era de $\phi 266 . \mathrm{kg}^{-1}$ de leche $(\$ 0,48)$.

De acuerdo con los registros del programa DairyProfit el ingreso principal de la finca proviene de las ventas de leche $(88,1 \%)$, otros ingresos provienen de los excedentes en forma de dividendos y premios que son entregados por la empresa industrializadora $(10,1 \%)$ y venta de animales (1\%). En los egresos el rubro más importante corresponde a la alimentación con un 51,1\%, seguido de la mano de obra $(26,7 \%)$ y transportes $(7,6 \%)$, este último contabiliza el costo del traslado de la leche a la planta procesadora.

\section{Capacidad de endeudamiento de la empresa}

Las razones de endeudamiento encontradas para la empresa se muestran en el Cuadro 3.

La razón de deuda mide el porcentaje de financiamiento aportado por los acreedores en la empresa y valores superiores al 70\% se consideran relativamente altos y las entidades financieras normalmente exigen niveles máximos de endeudamiento permitido. La razón pasivoaportación igualmente indica la proporción de la deuda asumida por los acreedores y valores bajos hacen a una empresa atractiva para obtener

Cuadro 3. Razones de endeudamiento para 3 períodos fiscales.

\begin{tabular}{lccc}
\hline & & Año \\
Razones de endeudamiento & 1 & 2 & 3 \\
\hline Razón de deuda ((pasivo total/activo total) x 100) & 27,29 & 10,73 & 13,41 \\
Razón pasivo-aportación ((pasivo total/capital total) x 100) & 37,53 & 12,02 & 15,49 \\
Cobertura de interés (utilidad operativa/gastos financieros) & 0,55 & 1,64 & 1,64 \\
\hline
\end{tabular}


financiamiento externo. La cobertura de intereses refleja la capacidad de la empresa para pagar y cubrir la carga financiera del período con sus utilidades (Salas 2005). La empresa mostró una tendencia a disminuir de forma significativa sus deudas y a contar con utilidades que permiten cubrir los gastos financieros eventualmente lo cual es una ventaja para ser sujeto de crédito ante entidades financieras externas para ampliar la capacidad productiva a mediano plazo.

\section{Proyección de la empresa bajo el sistema actual de producción}

Bajo el sistema actual solo se consideró como inversiones el sistema de manejo de las aguas de lavado y la remodelación de la sala de ordeño, las cuales en conjunto tienen un costo aproximado de $\$ 10.000 .000$, éstas son necesarias de realizar sin importar si se realiza o no la ampliación en capacidad productiva de la finca debido a la legislación ambiental y a razones de facilidad en la operación respectivamente. La sala de ordeño permitiría contar con un ahorro en el tiempo de espera de los animales previo al ordeño y una vez terminado, con lo cual el recurso mano de obra se podría utilizar para otras labores; además en el sistema actual se podría aumentar el tiempo de permanencia de los animales en el potrero y su consumo de materia seca del pasto lo cual se ha visto tiene un efecto directo sobre la productividad de las fincas de ganado de leche (Tozer et al. 2003).

Para proyectar el precio de la leche se utilizaron los precios históricos que ha recibido la empresa durante los últimos 4 períodos fiscales y se estimó la tendencia de cambio por medio de una regresión lineal y su respectiva fórmula de ajuste, a partir de la cual se proyectaron los precios de pago de la leche para los 10 años de análisis financiero (Figura 2).

Con base en las estimaciones del precio de la leche, se debe tener presente que de noviembre de 2008 a febrero de 2009 el precio internacional de la leche bajó de manera significativa y en general todas las expectativas y predicciones alrededor del mercado lácteo disminuyeron y cambiaron las expectativas que se tenían en el momento (Comisión de las Comunidades Europeas 2009). Luego de la situación de crisis atravesada, el panorama parece ser de nuevo alentador con posibilidades de crecimiento a nivel internacional donde se espera un crecimiento sostenido en la demanda de lácteos debido a las economías emergentes (Barquero 2011).

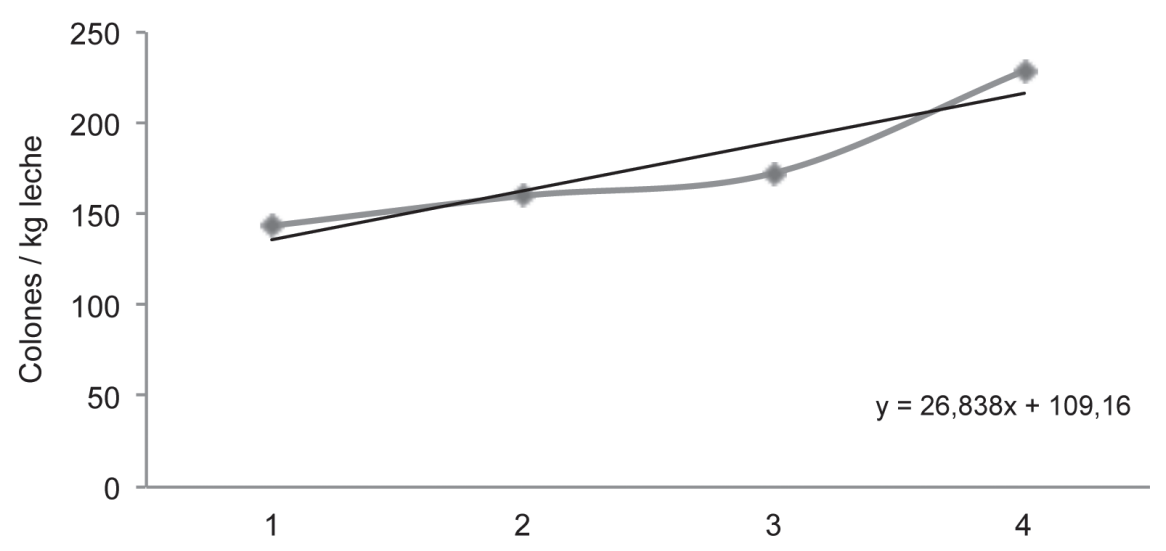

Fig. 2. Tendencia histórica en el precio de la leche para la empresa durante 4 años. 
Cuadro 4. Variación interanual según el índice de precios al consumidor de los últimos 10 años.

\begin{tabular}{cc}
\hline Años & Variación interanual \\
\hline $1998-1999$ & 9,92 \\
$1999-2000$ & 10,52 \\
$2000-2001$ & 10,97 \\
$2001-2002$ & 9,89 \\
$2002-2003$ & 8,91 \\
$2003-2004$ & 13,31 \\
$2004-2005$ & 13,94 \\
$2005-2006$ & 10,09 \\
$2006-2007$ & 9,84 \\
$2007-2008$ & 16,3 \\
Promedio & 11,37 \\
\hline
\end{tabular}

Para estimar la inflación en la proyección de los diferentes escenarios a evaluar en el proyecto, se tomaron las variaciones interanuales del índice de precios al consumidor de los últimos 10 años y se calculó el promedio, cifra que se utilizó como inflación esperada en el horizonte del proyecto (Cuadro 4) (INEC 2008a).

Con base en las estimaciones realizadas a la finca bajo su operación actual, tendría un aumento sostenido de los ingresos totales, sin embargo esta tendencia es seguida por los egresos y el aumento comparativo es mayor. Con base en el horizonte de evaluación de 10 años el VAN de la operación es de $\phi 147.960 .454$, con una inversión de $ф 10.000 .000$ (Cuadro 5).

Cuadro 5. Valor Actual Neto Incremental con base en la situación actual y las proyecciones de la ampliación en la capacidad productiva.

\begin{tabular}{lcc}
\hline Situación & VAN & VAN Incremental \\
\hline Actual & $\phi 147.960 .454,31$ & $\ldots$ \\
Inversiones sin compra de certificados & $\phi 237.129 .530,15$ & $\phi 89.169 .075,85$ \\
Inversiones con compra de certificados (Normal) & $\phi 14.278 .894,68$ & $-\phi 133.681 .559,62$ \\
Inversiones con compra de certificados (Optimista) & $\phi 74.092 .280,06$ & $-\phi 73.868 .174,24$ \\
Inversiones con compra de certificados (Pesimista) & $-\phi 45.534 .490,70$ & $-\phi 193.494 .945,00$ \\
\hline
\end{tabular}

\section{Situación técnica con el sistema de confinamiento}

Con la implementación del sistema de confinamiento, la finca experimentará cambios en cada una de las áreas funcionales debido a la ampliación en capacidad productiva y ciertas consideraciones que se debe tener en cuenta como referencia, el balance del análisis FODA realizado en la explotación.

\section{Producción}

Debido al menor gasto energético de los animales, se podría esperar un aumento en la producción ya que se suprime el gasto durante el pastoreo y de recorrido a la sala de ordeño, se estimó que este cambio podría generar en promedio hasta $2 \mathrm{~kg}$.animal ${ }^{-1} \cdot \mathrm{dí}^{-1}$ de leche, sin embargo esto se consideró en el análisis de riesgo de la situación propuesta con los escenarios optimista $(+2 \mathrm{~kg})$ y pesimista $(-2 \mathrm{~kg})$ (Tozer at al. 2003).

Se espera que haya además de un aumento individual, un aumento en la entrega total de leche pues el objetivo del productor es en ese período de 10 años llegar a 100 vacas en ordeño con base en el pie de cría de la finca. De acuerdo con la proyección de hato realizada, 
en el año 4 se alcanzaría alrededor de 75 vacas en ordeño lo cual, con las estimaciones llevadas a cabo, le permite al productor un flujo de caja adecuado para realizar inversiones como un corral nuevo.

Otra de las inversiones significativas que deberá realizar la finca es un carro mezclador para la repartición del alimento en forma de ración total mezclada (RTM), de esta forma se puede adicionar los suplementos mezclados con el pasto y repartir alimento de forma más eficiente y constante tal como lo requieren los animales en sistemas estabulados. Eventualmente se pueden tener ventajas en términos productivos desde el punto de vista de balance de raciones propias y compra de materias primas de forma directa que pueden ajustarse a los requerimientos del sistema productivo.

\section{Recursos humanos}

La empresa no considera dentro de los cambios operativos ampliar la planilla actual de trabajadores, sin embargo se debe realizar una adecuación de los puestos de trabajo y la asignación de labores que permitan mantener el funcionamiento del sistema con los cambios operativos implementados. Una de las conclusiones del análisis FODA que se realizó en la finca es que se debe buscar una mayor independencia del gerente para la realización de las labores diarias, es por eso que se propone un programa de capacitación que sea inclusivo de todo el personal con el objetivo de que cada trabajador cuente con las herramientas que les permitan ser más versátiles dentro de la finca.

\section{Mercadeo}

De acuerdo con las condiciones predominantes en el momento en que se realizó el estudio, se consideraba que el mercado lácteo internacional y nacional estaría abierto a la entrega de leche por al menos 5 años debido al déficit que tenían muchos de los principales países importadores de leche, a través del ingreso de nuevos socios a las plantas o del aumento en producción de los socios actuales por medio de los mecanismos y facilidades como la compra de certificados de aportación para la entrega de la leche. Este último aspecto es incierto pues se trata de la incertidumbre inherente a las políticas de una empresa y no una regulación generalizada.

Uno de los escenarios propuestos fue la ampliación en capacidad productiva a través del pago diferenciado de la leche, con un pago del 90\% del precio. De acuerdo con las tendencias en el mercado, esa situación, no debería ser un problema para el crecimiento sostenido que piensa llevar a cabo la empresa, ya que puede prepararse para el futuro y negociar una mayor entrega de leche a la planta.

\section{Situación financiera con el sistema de confinamiento}

\section{Inversiones significativas}

Dentro de las inversiones necesarias para la implementación del sistema estabulado se encuentran en orden de importancia: compra de certificados de aportación, construcción de un corral nuevo con materiales y estructuras disponibles en la finca, compra de un carro mezclador de alimento, implementación de un sistema de manejo y riego de las aguas de lavado y la modificación de la sala de ordeño actual.

En el caso de los certificados de aportación se consideró el ajuste en precio que realizó COPROLE R.L. en octubre de 2008 y cuya adquisición se llevaría a cabo de forma gradual con base en las proyecciones realizadas en el programa Invest-GA®.

El corral nuevo debería construirse en el año 3, para que esté listo en el año 4 donde se da un aumento significativo en el tamaño del hato en producción, asimismo el carro mezclador se comprará en el tercer año con el mismo objetivo y que haya una repartición más ágil del alimento en el estabulado. 


\section{Proyección del sistema estabulado con inversiones y sin compra de certificados de aportación}

Como se mencionó anteriormente, uno de los escenarios planteados fue el crecimiento de la finca únicamente por medio de la inversión en un corral, el carro mezclador además del pago diferenciado de la planta industrial (alrededor de 90\% del precio nominal) (Cuadro 5). En el análisis financiero para dicho escenario se pudo observar como los ingresos y los egresos operativos totales muestran incremento, aunque éstos últimos reportaron un crecimiento menor. El VAN obtenido fue de $\phi 237.129 .530,15$ con un monto de inversión de $ф 53.063 .072$.

\section{Análisis de sensibilidad del sistema estabulado con inversiones y compra de certificados (escenarios)}

Para el análisis de sensibilidad se escogió la situación en la cual la empresa realizaría una inversión mayor (corral, carro mezclador y compra de certificados de aportación) por un monto de $\phi 201.263 .479,49$. En el Cuadro 6 se muestra el resumen de los montos obtenidos para la utilidad neta para el décimo año y el VAN en cada uno de los escenarios propuestos.

El análisis de sensibilidad permite evidenciar desviaciones en los valores de las variables con base en efectos de consideración que pueden afectar la medición de los resultados (Sapag y Sapag 2008). En el caso de las fincas de ganado de leche, el grado de liquidez que opera es determinante para el desempeño financiero, ya que por medio de los pagos semanales se pueden realizar compras de insumos además de negociar precios y realizar inversiones o reinversiones de menor magnitud pero necesarias en la operación.

La situación optimista y normal reportaron un VAN positivo mientras la situación pesimista mostró un valor negativo; este último escenario podría presentarse, entre otras razones, debido a factores de tipo ambiental y el estrés en los animales y por consiguiente, una caída en la producción de leche resultado del cambio. Sapag y Sapag (2008) mencionan que las proyecciones pueden variar en su resultado final debido a lo inciertas que son algunas variables incontrolables como cambios en los niveles de los precios reales del producto o de los insumos.

\section{Valor Actual Neto Incremental}

Con base en el Cuadro 5 se puede observar que la proyección en la que se invierte en el corral de estabulado y el carro mezclador sin adquirir certificados de aportación, es donde se obtuvo el VAN nominal e incremental superior con respecto a los demás escenarios. En el caso de que se adquieran certificados, el escenario normal reportó un VAN nominal inferior al de la situación actual proyectada y por tanto un VAN incremental negativo por lo que desde ese punto de vista es preferible mantener el sistema de producción actual y no es factible llevar a cabo las inversiones para ampliar la capacidad en producción.

El escenario optimista mostró un VAN nominal inferior al de la situación actual y sin adquirir certificados, en este caso igualmente se ve que para la finca es preferible seguir bajo el modelo actual antes de realizar una inversión

Cuadro 6. Utilidad neta al décimo año y Valor Actual Neto bajo diferentes escenarios de sensibilidad.

\begin{tabular}{ccc}
\hline Situación bajo compra de certificados & VAN & Utilidad neta (10 ${ }^{\text {mo }}$ año $)$ \\
\hline Normal & $\phi 14.278 .894,68$ & $\phi 37.668 .586,91$ \\
Optimista & $\phi 74.092 .280,06$ & $\phi 56.676 .232,67$ \\
Pesimista & $-\phi 45.534 .490,70$ & $\phi 18.660 .941,16$ \\
\hline
\end{tabular}


tan alta pues, aún con condiciones favorables el endeudamiento de la empresa disminuye su liquidez. Por último la situación pesimista reportó el VAN nominal más bajo de los 4 escenarios y por tanto el VAN incremental con el valor más negativo el cual es todavía menor al escenario normal con compra de certificados.

\section{CONCLUSIONES Y RECOMENDACIONES}

La empresa cuenta con una liquidez y un flujo de caja tal que le permite mayor capacidad de endeudamiento y acceso al financiamiento externo para aumentar su capacidad productiva.

Las inversiones necesarias para aumentar la capacidad productiva de la finca, pueden realizarse de forma gradual lo que permitiría que no haya una disminución marcada en la liquidez debido al endeudamiento con las entidades financieras. El sistema de manejo de aguas de lavado puede generar beneficios adicionales en el manejo de las pasturas y ahorro en la compra de insumos para la finca, la sala de ordeño disminuiría el tiempo de espera de los animales lo que permite más tiempo de consumo de pasto en potrero o en corral y menos tiempo en la labor de ordeño por parte del personal, el carro mezclador por su parte permitiría el ajuste de las raciones y una mayor eficiencia en la repartición del alimento a los animales en el corral.

La implementación de un sistema de estabulación total implica inversiones que deben compensarse con un aumento en la producción ya sea individual $\left(\mathrm{kg} \cdot \mathrm{vaca}^{-1}\right.$.día $^{-1}$ de leche) o total (aumento en el tamaño del hato y la carga animal), que permitan mantener un nivel de utilidad adecuado mientras se cancelan los gastos financieros. Esto va a depender también de las condiciones de cada finca y del criterio del productor.

El análisis financiero realizado en los diferentes escenarios mostró que la alternativa más viable para la implementación del sistema de producción estabulado es invertir en la construcción de un corral y la compra de un carro mezclador sin adquirir certificados de aportación. En caso de ejecutarse un proceso de aumento en la capacidad productiva, deben considerarse las variaciones inherentes al mercado lácteo nacional e internacional que pueden modificar el panorama de inversión.

El costo de producción del kg de leche es determinante de la competitividad de la finca y se debe considerar no sólo el hecho que sea menor al pago de la leche, sino también el volumen requerido para compensar los costos marginales que genera un cambio en el sistema productivo.

\section{AGRADECIMIENTOS}

Los autores expresan su agradecimiento al propietario de la finca donde se realizó esta investigación: Ing. Alvaro Coto, así como al Ing. César Solano de la empresa IAP-Soft por la colaboración brindada para la realización de la investigación.

\section{LITERATURA CITADA}

BARQUERO M. 2011. Exportación de productos lácteos tendrá década de auge. Artículo del periódico La Nación. Lunes 6 de junio del 2011. Costa Rica. 30A p.

CAMPOS W. 2005. Evaluación técnica y financiera de los sistemas de producción: pastoreo, semiestabulado y estabulado completo en lechería especializada. Tesis de licenciatura, Universidad de Costa Rica, San José, Costa Rica. 81 p.

COMISIÓN DE LAS COMUNIDADES EUROPEAS. 2009. Situación del mercado de la leche y los productos lácteos en 2009. Comunicado de la Comisión al Consejo. Bruselas, $15 \mathrm{p}$.

DELGADO J. 2008. Propuesta estratégica y operacional para la implementación de una unidad productiva dedicada a la actividad caprina ubicada en El Llano, San Miguel, Desamparados, San José. Tesis de maestría, Universidad de Costa Rica, San José, Costa Rica. 85 p.

IAP SOFT. 2007. DairyProfit: herramienta para el monitoreo y evaluación del desempeño técnicoeconómico de fincas lecheras bajo un esquema sencillo y estándar. Programa de computo parte del paquete InforDairy, versión 2.0. IAP SOFT. Cartago, Costa Rica.

IAP SOFT. 2008. Invest-GA: Sistema para la evaluación de inversiones en Ganadería. Programa de computo, versión 1.0. IAP. SOFT. Cartago, Costa Rica. 
INEC 2008a. Índice de precios al consumidor base julio 2006. Volumen 10, año 19. 4 p.

JANZEN D.H. 1991. Historia Natural de Costa Rica. $1^{\text {a }}$ ed. Editorial de la UCR, San José, Costa Rica. 822 p.

RETANA J. 2011. Climatología de la región del distrito de Santa Rosa de Oreamuno en base a estaciones meteorológicas cercanas a la zona. Informe anual del Instituto Meteorológico Nacional. 3 p.

SALAS T. 2005. Análisis y diagnóstico financiero: enfoque integral. $4^{\text {ta }}$ ed. Ediciones Guayacán, San José, Costa Rica. $340 \mathrm{p}$

SAPAG N., SAPAG R. 2008. Preparación y evaluación de proyectos. $5^{\text {ta }}$ ed. McGraw-Hill Interamericana S.A. México. 445 p.

SERVICIO NACIONAL DE SALUD ANIMAL. 2006. Lineamientos generales para la implementación y otorgamiento de Certificados Veterinarios de
Operación al amparo de la Ley 8495 del 6 de abril del 2006 (Ley SENASA). San José, Costa Rica. 54 p.

TOZER P.R., BARGO F., MULLER L.D. 2003. Economic Analyses of Feeding Systems Combining Pasture and Total Mixed Ration. Journal of Dairy Science 86:808-818.

VASQUEZ N. 2011. Producción de ovinos de carne en Costa Rica: Estudio de factibilidad para la implementación de un modelo productivo en Ciudad Quesada. Tesis de licenciatura, Universidad de Costa Rica, San José, Costa Rica. 303 p.

VILLEGAS L. 2007b. Factores determinantes para la productividad de fincas lecheras en pastoreo. Análisis de casos en fincas de bajura. Revista ECAG Informa. No. 39:17-19. Balsa de Atenas, Costa Rica. 